\title{
R-spondins are Expressed by Ewing sarcoma Cells and are Potential Targets for Metastasis Inhibition.
}

Elisabeth A. Pedersen, BS; Morgan K. Penny, BS; Raelene A. Van Noord, BS, MS; Mark J. Hoenerhoff DVM, PhD, DACVP; and Elizabeth R. Lawlor MD, PhD

\section{ABSTRACT}

\section{Background}

Ewing sarcoma is a highly metastatic tumor, and the Wnt/ beta-catenin signaling pathway was recently shown to contribute to the metastatic phenotype. R-spondin (RSPO) ligands potentiate canonical Wnt signaling in human cancers, including Ewing sarcoma cells, by binding to the LGR family of receptor proteins. However, the pattern of RSPO expression in Ewing sarcoma has not previously been defined.

\section{Methods}

Author E-mail List: easp @med.umich.edu; mkpenny @umich.edu; raelenec@umich .edu; hoenerho@med.umich .edu; elawlor@med.umich.edu

Corresponding Author: Elisabeth A.Pedersen, BS, easp@med .umich.edu, 248-719-3051

\section{Conflicts of interest: The} authors have no conflicts of interest to disclose. well as metastasis were examined using in vivo xenograft models.

\section{Results}

RSPOs are expressed by Ewing sarcoma cells and tissues that are frequent sites of metastasis. OMP130-M230 had moderate ability to block RSPO signaling in vitro. In vivo, inhibition of RSPO2 did not affect tumor size, but significantly inhibited the frequency of metastatic nodule formation.

\section{Conclusions}

RSPOs and associated signaling molecules are expressed by Ewing sarcoma cells themselves and also by tissues to which Ewing sarcoma metastasizes. Inhibition of RSPO2 blunts Wnt/beta-catenin signaling response in vitro and reduces formation of metastasis. These studies provide preliminary evidence that RSPOs may be amenable to therapeutic targeting for metastasis inhibition in Ewing sarcoma. 


\section{Introduction}

E wing sarcoma is an aggressive tumor that most commonly affects adolescents, but can occur in patients of any age. It is the second most common bone tumor, most frequently occurring in the long bones of patients, but also can present as soft tissue tumors. Its current treatment regimen is intensive and the regimen consists of five chemotherapies (vincristine, doxorubicin, cyclophosphamide, irinotecan, and etoposide) along with surgery and radiation. Despite this multimodal and toxic therapy, up to $30 \%$ of patients experience metastasis and/or relapse, which results in a poor prognosis. ' Ewing sarcoma tumors are characterized by pathognomonic chromosomal translocations resulting in EWS/ETS fusion proteins, the most common of which is the $t(11 ; 22)$ translocation resulting in an EWS/FLI-1 fusion protein. ${ }^{2}$ EWS/ETS fusion proteins are required for tumor growth and proliferation, but the mechanisms that contribute to Ewing sarcoma metastasis remain largely unknown. Recent evidence implicates the Wnt/beta-catenin signaling pathway as a contributor to metastasis in Ewing sarcoma. ${ }^{3-7}$ The Wnt/beta-catenin signaling pathway is required for normal embryogenesis and adult tissue homeostasis and is frequently dysregulated in cancer. ${ }^{8}$ In Ewing sarcoma, stimulation with canonical Wnt ligands activates the Wnt/beta-catenin signaling pathway and causes a switch from a proliferative state to a migratory and invasive state by antagonizing the growthpromoting signals of EWS/ETS proteins and altering the tumor cell cytoskeleton. ${ }^{3}$ As a result, the Wnt/beta-catenin signaling pathway has recently emerged as a possible avenue for therapeutic intervention.

In Ewing sarcoma, as well as other tumor types, activation of Wnt/beta-catenin signaling can be potentiated by the R-spondin (RSPO) family of molecules. ${ }^{4,9}$ RSPOs are a family of four molecules, known as RSPO1-4, which are secreted ligands that bind to LGR4, LGR5, and LGR6 members of the leucine-rich repeat containing G-protein coupled receptor (LGR) family of receptors. In the absence of Wnt ligands, RSPOs have no activity and do not affect Wnt signaling. However, when Wnt ligands are present at the cell membrane, binding of RSPO ligands to LGR receptors inhibits downstream E3-ubiquitin ligase family members ZNRF3 and RNF43. ZNRF3 and RNF43 function to ubiquitinate Wnt receptors, which results in degradation of the Wht receptor complex. ${ }^{9}$ RSPO/LGR signaling inhibits this degradation and thus stabilizes the Wnt receptor complex at the cell membrane. This in turn results in increased and sustained Wnt signaling activity. ${ }^{9}, 10$

RSPOs have been implicated as mitogenic factors that promote tumor growth and development 9,11,12; however, their contribution to the metastatic process remains largely unexplored. In Ewing sarcoma, we found that stimulation with exogenous RSPO2 results in an increased metastatic phenotype. ${ }^{3}$ In addition, Anti-RSPO antibodies have recently been developed for therapeutic applications in anticancer treatment regimens for numerous cancers, including colorectal and breast carcinomas. ${ }^{13}$ Thus, it was our hypothesis that RSPOs are secreted by Ewing sarcoma tumors and function to promote Ewing sarcoma metastasis. In this study, we sought to further characterize RSPO expression and function in Ewing sarcoma cells and to determine if inhibition of RSPO should be explored as a potential antimetastatic therapy.

\section{Methods}

\section{Cell culture and lentiviral transductions}

Ewing sarcoma cell lines A673, CHLA25, CHLA32, STA-ET-8.2, TC71, and TC32 cells were cultured in RPMI 1640 media (Gibco, Waltham, MA) supplemented with 10\% fetal bovine serum (FBS) (Atlas Biologicals, Fort Collins, CO) and 2mM L-glutamine (Life Technologies, Carlsbad, CA). CHLA25, CHLA32, and STA-ET-8.2 were grown on plates coated with $0.2 \%$ gelatin. Wnt3a and control media were derived from L-cells (ATCC CRL-2648) and Wnt3a 
L-cells (ATCC CRL-2647) cultured in DMEM (Gibco Waltham, MA) supplemented with 10\% FBS. Identities of the cells were verified by short tandem repeat (STR) profiling. Cells were routinely tested for mycoplasma contamination and were verified to be negative for all studies. Lentiviral production and transduction were performed as previously described. ${ }^{4}$

\section{Gene expression assays}

To determine if Ewing sarcoma cells express RSPOs, we used qRT-PCR to determine the level of expression of each RSPO gene in six Ewing sarcoma cell lines. Although Ewing sarcoma cells secrete RSPOs, their expression is highly variable, leading us to question whether RSPO ligands might also be provided by non-tumor cells in the surrounding microenvironment. To address this, we sought to determine if cells present in the tissues harboring Ewing sarcoma metastases in xenograft models express RSPOs. Primary tissues were isolated from wild-type mice, and expression of RSPO family members was measured in liver, lung, bone marrow, and intestine.

RNA was extracted from cell lysates using the Qiagen RNA extraction kit (Qiagen, Hilden, Germany), either from cell lines or from tissues dissected from wild-type NOD SCID mice (strain 394, Charles River Laboratories, Wilmington, MA). Sections of mouse tissue that were $1 \times 1 \mathrm{~mm}$ were flash frozen in liquid nitrogen immediately following dissection, then thawed and homogenized in Qiagen lysis buffer using an $18 \mathrm{~g}$ needle to make cell lysates. cDNA was generated from RNA using the iScript kid (Bio-Rad, Hercules, CA), and qRT-PCR was performed with primer sequences designed using qPrimerDepot (primerdepot.nci.nih.gov) (Table 1) and validated using positive controls. Assays were performed in triplicate using the Roche Light Cycler 480, and average Ct values were normalized relative to the housekeeping genes hypoxanthineguanine phosphoribosyltransferase 1 (HPRT1) or ribosomal subunit 18 s expression in the same sample. Per standard convention, human genes are indicated by all uppercase letters in italics, while mouse genes are identified by lowercase letters in italics.

\section{Assessment of OMP130-M230 activity}

Having previously established that RSPO2, in particular, potentiates Wnt/beta-catenin signaling in Ewing sarcoma to promote metastatic disease, we next sought to determine the impact of blocking RSPO2. To achieve this, we obtained the mouse monoclonal antibody OMP130-M230, developed and kindly provided by Oncomed Pharmaceuticals (Redwood City, CA), ${ }^{13}$ and tested the ability of this antibody to block RSPO2-dependent signaling in vitro and tumor growth in vivo. First, to determine if the antibody effectively blocks RSPO2 signaling in Ewing sarcoma cells in vitro, we used a Ewing sarcoma cell line that has been engineered to express a green fluorescent protein-based (GFP-based) reporter of Wnt/beta-catenin activity. To generate these cells, termed A673-7TGP, A673 cells were transduced with plasmid \#24305 (Addgene, Cambridge, MA). (A673 cells were used given their high efficiency of plasmid incorporation.) To test if OMP130-M230 can block RSPO2mediated potentiation of GFP expression, we stimulated cells with Wnt3a CM +/- RSPO2 in the presence of $100 \mathrm{mg} / \mathrm{mL}$ antibody or saline control. Stably transduced A673-7TGP cells were stimulated with 1:1 RPMI1640 supplemented with 5\% FBS and 1\% L-glutamine and conditioned media (CM) from control-L-cells or Wnt3a-L-cells +/- RSPO2 for 48 hours, with either saline control or $100 \mu \mathrm{g} / \mathrm{mL}$ antibody. Cells were dissociated using Accutase (Millipore, Burlington, MA), and fluorescence was measured using an Accuri C6 cytometer.

\section{Subcutaneous tumor growth}

Tumor xenografts were established using two Ewing sarcoma cell lines, A673 and TC32, by subcutaneously injecting tumor cells in Matrigel ( $n=8$ mice per cell line per condition). A673 and TC32 cell lines were chosen for their high efficiency of subcutaneous tumor formation. ${ }^{14}$ In order to assess the efficacy of the antibody 
TABLE 1. Primer Sequences Used for qRT-PCR.

\section{Gene}

hu HPRT1 fwd

hu HPRT1 rev

hu RSPO1 fwd

hu RSPO1 rev

hu RSPO2 fwd

hu RSPO2 rev

hu RSPO3 fwd

hu RSPO3 rev

hu RSPO4 fwd

hu RSPO4 rev

ms 18s fwd

ms 18 s rev

ms Rspo1 fwd

ms Rspo1 rev

ms Rspo2 fwd

ms Rspo2 rev

ms Rspo3 fwd

ms Rspo3 rev

ms Rspo4 fwd

ms Rspo4 rev

\section{Sequence}

TGAACACTGGCAAAACAATGCA

GGTCCTITICACCAGCAAGCT

TGGAGAGGAACGACATCCG

CCTTACACTTGGTGCAGAAGTTA

GACGCAGTAAGCGAGCTAGTT

ACATCGGCTACACCCATTGTC

TGTGCAACATGCTCAGATTACA

TGCTTCATGCCAATTCTTTCCA

ACAGGCTGTATCATCTGCTCA

GATGCCGAAGTACCCAGGG

GCAATTATTCCCCATGAACGA

GGCCTCACTAAACCATCCAAT

GGGATCAAGGGCAAGAGACAG

CTGGCGGATGTCGTTCCTC

CCAAGGCAACCGATGGAGAC

TCGGCTGCAACCATTGTCC

ATGCACTTGGCGACTGATTTCT

GCAGCCTTGACTGACATTAGGAT

CTCGCCCTGTACCGAAGGA

CACTTGCCGTACTGACGGATG as a therapeutic option in established tumors, cells were first allowed to engraft and form small tumors. Once tumors were established, weekly intraperitoneal (IP) administration of antibody was started. 1×106 A673 or TC32 cells were re-suspended in Matrigel and RPMI media (1:1) and subcutaneously implanted into 10- to 12-week-old NOD SCID mice (strain 394, Charles River Laboratories, Wilmington, MA). For each cell line, $n=8$ mice were used per condition. Tumors were measured every other day by calipers, and tumor volume was determined using the formula
$V=1 / 2 a b 2$, where $a$ indicates the longer dimension and $b$ indicates the shorter dimension. Once tumors reached $200-500 \mathrm{~mm}^{3}$, weekly administration of the anti-RSPO2 monoclonal antibody was administered intraperitoneally at a dose of $20 \mathrm{mg} / \mathrm{kg}$ or an equal volume of saline as a control. Mice were euthanized once tumors reached $18 \mathrm{~mm}$ in any one dimension.

\section{In vivo subrenal capsule metastasis model}

Next, we evaluated whether RSPO2 inhibition impacts tumor metastasis. Since Ewing sarcoma cell 
lines do not effectively metastasize from subcutaneous tumors, we utilized a subrenal capsule model as a method to assess the full metastatic cascade. ${ }^{14}$ TC32 cells were used because they have a high rate of metastasis compared to other Ewing sarcoma cell lines. ${ }^{14}$ In this study, luciferase-tagged TC32 cells were directly injected under the capsule of the kidney via ultrasound-guided needle and catheter. A primary tumor was established in the kidney, which is a highly vascularized organ, thus providing ample opportunity for tumor cells to access the bloodstream. Weekly IP injection of anti-RSPO2 antibody was started at the time of tumor implantation. Primary tumor growth and appearance of metastases were monitored by bioluminescence imaging. To generate luciferase-tagged cells, TC32 cells were transduced with pLentilox-luciferase/ GFP (UM Vector core) for luciferase-tagged cells and then selected in puromycin. ${ }^{15}$ As previously described, 2×105 luciferase-tagged TC32 cells were directly injected under the capsule of the kidney via ultrasound-guided needle and catheter. ${ }^{14}$ Weekly IP injections of 20 mg/kg OMP130-M230, or an equal volume of saline, was started at the time of tumor implantation ( $n=10$ mice per condition). Primary tumor growth and the appearance of large metastases were followed weekly via bioluminescence imaging. After six weeks, each mouse was first injected with luciferin and the primary tumor was imaged. Mice were then euthanized 5 minutes after luciferase injection, and their kidneys containing the primary tumor were removed via dissection. Immediately following kidney dissection, the remaining organs were imaged by bioluminescence ex vivo, and the number of overt metastases, as well as the bioluminescent tumor burden of distant metastases was measured via bioluminescence and macroscopic visualization with the aid of ULAM-PCAR. The presence of metastases was microscopically confirmed by histopathology.

\section{Statistical analysis}

Data for gene expression levels, tumor volume, bioluminescent signal, and number of metastases are expressed as mean \pm standard error of the mean (SEM), calculated using GraphPad Prism. Significance was determined using the Student's t-test, and p-values $<0.05$ were considered significant. T-test analysis was performed GraphPad Prism software.

\section{Results \\ $R$-spondins are expressed by both Ewing sarcoma cells and metastatic tissue sites}

In a panel of six Ewing sarcoma cell lines, we found that none of the cell lines expressed RSPO1, all cell lines expressed RSPO2 (approximately $0.1 \%$ HPRT in TC32) and RSPO3, and five of six lines expressed RSPO4 (Figure 1A). In addition, we measured expression of RSPO receptors and LGR family members 4, 5, and 6. All cell lines expressed LGR4 and LGR5, but no cell lines demonstrated $L G R 6$ expression (Figure 1B). To determine if Ewing sarcoma cells have all of the components required for RSPO signaling, we also measured expression of E3 ubiquitin ligases RNF43 and ZNRF3. We found that all cells express both genes, although ZNRF3 expression was on average higher than RNF43 expression (Figure 1C). These data demonstrate that Ewing sarcoma cells express all the components necessary for endogenous RSPO signaling. In regards to tissue expression, Rspo1, Rspo2, and Rspo3 were expressed by liver, liver, lung, and small intestinal tissue, with Rspo 1 having the most robust expression overall (Figure 2). Rspo4 expression was only detected in lung tissue.

\section{A monoclonal antibody against RSPO2 blocks Wnt/beta-catenin signaling}

As shown by flow cytometry, Ewing sarcoma cells do not display basal Wnt activity and thus do not express GFP (Figure 3A, left panel). GFP expression is induced in the presence of Wnt ligand, and this expression is further potentiated by the addition of exogenous RSPO2 (Figure 3A, middle and right panels). As shown, OMP130-M230 blunted RSPO2-mediated potentiation of GFP expression (Figure 3B). Having shown in vitro 


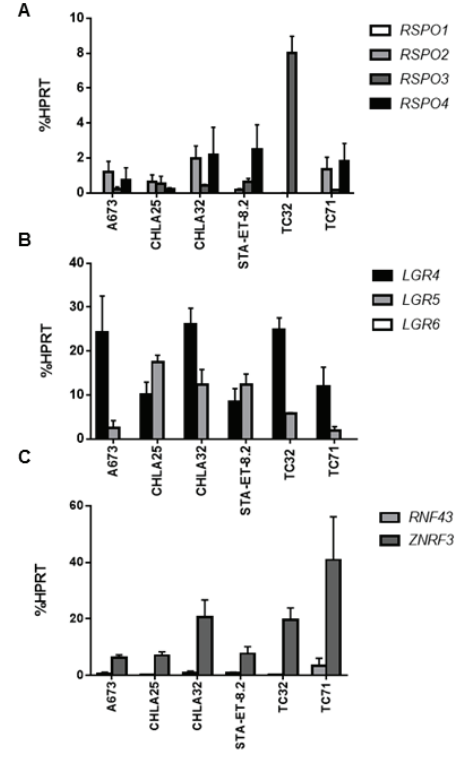

FIGURE 1. Components of the RSPO/LGR/RNF signaling pathway are expressed by Ewing sarcoma. (A) R-spondin genes (RSPO1-4), (B) LGR genes LGR4-6, and (C) RNF43, ZNRF3 are expressed to varying degrees by 6 different Ewing sarcoma cell lines. Gene expression was measured by $\mathrm{qRT}$-PCR and is shown relative to the housekeeping gene HPRT. Data is shown as mean of $\mathrm{n}=3$ independent replicates \pm SEM.

A
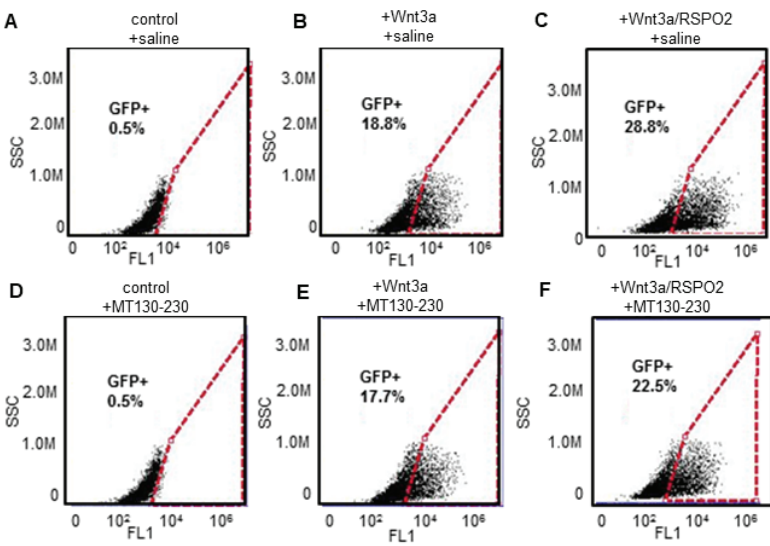

FIGURE 3. A monoclonal antibody against RSPO2 (OMP130-M230) blocks induction of RSPO-mediated Wht activity in vitro. A673 Ewing sarcoma cells were transduced with a GFP reporter of Wnt activity.

No GFP expressed under control conditions (A).

Addition of Wnt3a induces GFP (B), which is further increased by addition of exogenous RSPO2 (C).

Addition of OMP130-M230 does not affect GFP expression under control conditions (D) or Wnt3a conditions (E) but inhibits potentiation by exogenous RSPO2 (F).

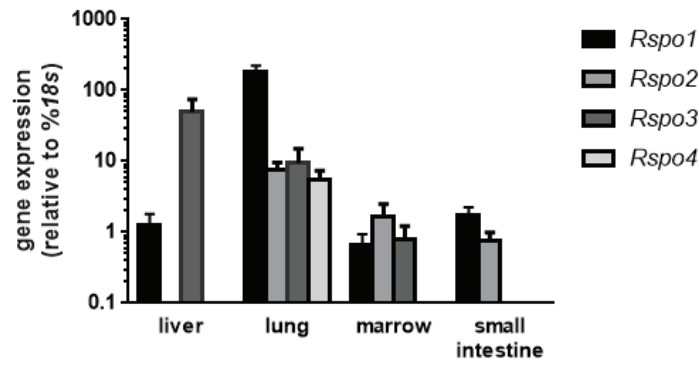

FIGURE 2. RSPOs are expressed to varying levels by sites to which Ewing sarcoma frequently metastasizes. Gene expression of Rspo 1-4 in mouse tissue is shown as measured by qRT-PCR expression relative to the housekeeping gene 18s. Data is shown as mean of $\mathrm{n}=3$ mice \pm SEM.

A

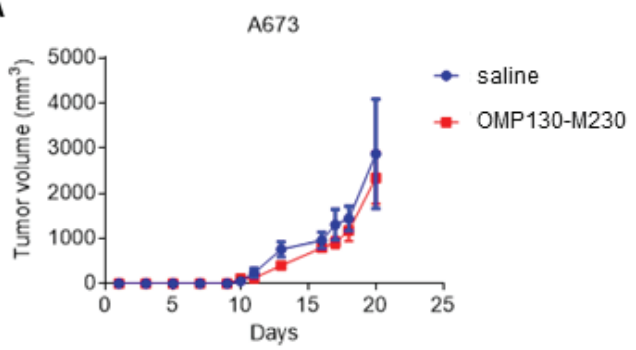

B

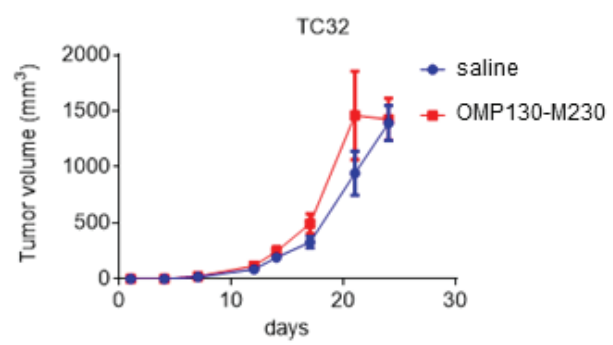

FIGURE 4. RSPO2 inhibition does not affect subcutaneous tumor growth.

(A) A673 and (B) TC32 cells were implanted subcutaneously, and weekly IP administration of OMP130-M230 was started once tumors reached $200-500 \mathrm{~mm}^{3}$ in volume. Tumor volume was measured over time and no significant differences were observed between groups. Data is shown as mean of $n=8$ mice per group \pm SEM. 
efficacy, we next tested the effects of this antibody on subcutaneous tumor growth. In xenografts of A673 (Figure 4A) or TC32 (Figure $4 B)$, there was no difference in the volume of tumors in mice treated with saline control or OMP130-M230.

\section{Inhibition of RSPO2 results in no growth differences but decreases the number of metastatic tumors}

Next, we evaluated whether RSPO2 inhibition impacts tumor metastasis. TC32 cells established large primary tumors in the kidney after six weeks (Figures 5A, 5B). We also demonstrated that, after the kidney was removed, the remaining organs were effectively imaged by bioluminescence ex vivo, and the number of overt metastases correlated with bioluminescence imaging (Figure 5C). Representative images of metastases detected ex vivo are shown in Figure 5D. Comparison of

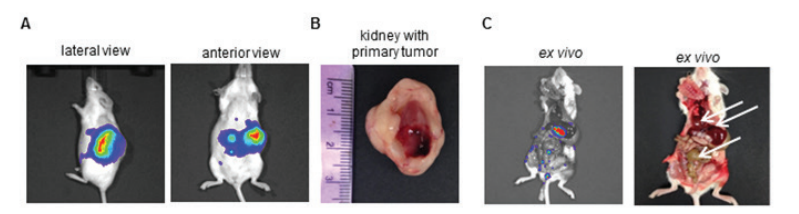

D
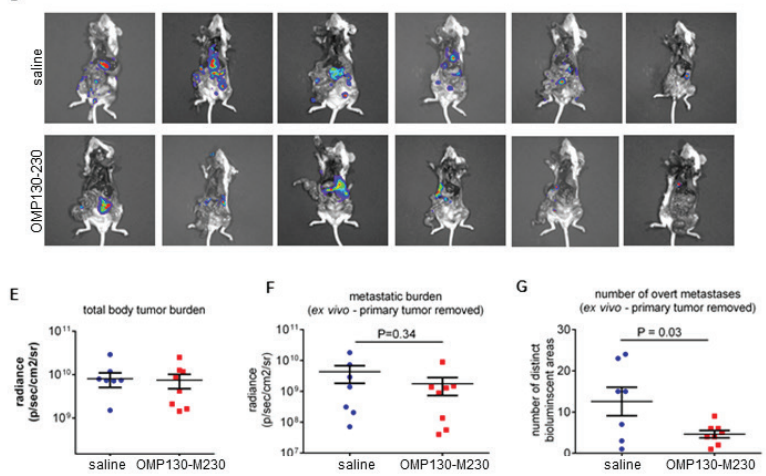

mice treated with saline or with antibody revealed that both groups efficiently formed large primary tumors, and there was no notable difference in primary tumor burden (Figure 5E). Likewise, upon primary tumor removal, all mice had evidence of abdominal dissemination or distant metastases, with a slight but not statistically significant decrease in overall metastatic burden in mice treated with OMP130-M230 (Figure 5F). However, the overall number of disseminated and metastatic tumor nodules was significantly reduced in antibody-treated mice (Figures 5D, 5G). Mice treated with saline had numerous tumor nodules throughout the body and especially in the gastrointestinal system, whereas mice treated with the anti-RSPO2 antibody had fewer nodules (although they were similar in size to those in the control mice).

The growth and engraftment of primary tumors in the subrenal capsule were confirmed

FIGURE 5. RSPO2 inhibition does not affect tumor burden but reduces the number of metastatic nodules in a model of metastasis.

(A) After ultrasound-guided injection into the subrenal space, luciferase-tagged TC32 cells formed primary tumors, and tumor growth was monitored by bioluminescence for six weeks.

(B) Representative image showing that primary tumors grew in and around the kidney. (C) After kidney and primary tumor were dissected out as in (B), mice were immediately re-imaged ex vivo, and metastases were detected by bioluminescent imaging (left panels) and confirmed as tumor nodules (arrows, right panel).

(D) Representative ex vivo images of tumor burden from mice treated with saline control (top panels) or OMP130-M230 (bottom panels). No difference was observed in overall primary (E) tumor or metastatic (F) tumor burden between groups. Data is expressed as radiance in each mouse and error bars indicate SEM.

$(G)$ Distinct areas of bioluminescent signal were counted and revealed that mice treated with OMP130-M230 had significantly less metastatic nodule formation than mice treated with saline. Data is expressed as number of nodules in each mouse and error bars indicate SEM. 


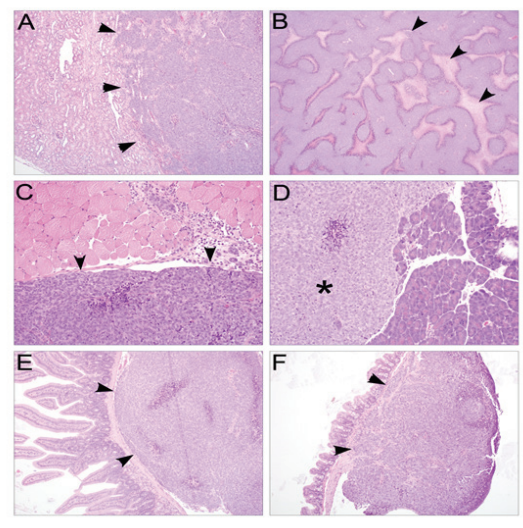

histologically (Figure 6A) and demonstrated areas of necrosis consistent with typical Ewing sarcoma growth patterns (Figure 6B). For both conditions, distant spread was observed in numerous sites, including the visceral surface of the diaphragm (Figure 6C), in the pancreas (Figure 6D), and on the serosal surfaces of the small intestine (Figure 6E) and colon (Figure 6F).

\section{Discussion}

These data provide evidence that R-spondin genes are expressed by both Ewing sarcoma cells and the tissues to which these cells metastasize. We demonstrated that the monoclonal antibody OMP130-M230 partially inhibits the action of RSPO2 on Wnt/beta-catenin activation in vitro. When assessing tumor growth using subcutaneous xenografts, inhibition of RSPO2 had no significant effect on tumor size. Similarly, there was a trend toward decreased bioluminescence signal of metastases in our metastasis model, suggesting that once metastases were established, there was a slight but not statistically significant difference of metastatic tumor growth. Despite little inhibition of tumor growth being observed, inhibition of RSPO2 significantly inhibited the number of metastatic nodules that were formed. These data support our hypothesis that RSPOs are expressed by Ewing sarcoma cells and provide evidence for a role in metastasis. Specifically, these data suggest
FIGURE 6: Representative images of primary xenografts and metastatic nodules confirm tumor engraftment and spread.

(A) Primary tumor xenografts were largely expansile and infiltrative, often effacing large portions of kidney (arrowheads), and were associated with large zones of serpentine necrosis ( $B$, arrowheads).

Spread within the abdominal cavity most often involved the visceral surface of the diaphragm (C, arrowheads), pancreas ( $D$, tumor nodule, asterisk) or peripancreatic tissues, serosal surfaces of the small intestine ( $E$, arrowheads), and colon ( $F$, arrowheads) with invasion into the underlying tunica muscularis.

that inhibition of RSPO2 via the OMP130-M230 antibody has little effect on the overall size of primary or metastatic Ewing sarcoma tumors in vivo, but it significantly reduces the ability of primary Ewing sarcoma tumors to seed distant organs.

RSPOs are important for normal regulation of the Wnt/beta-catenin signaling pathway and are becoming recognized as potential contributors to cancer progression. 11,12 Our previous work revealed a role for RSPO signaling as a molecule that amplifies Wnt/beta-catenin signaling activity, which in turn, promotes metastasis., ${ }^{3,4}$ The current work expands upon our previous findings and reveals that Ewing sarcoma cells themselves express RSPOs, as well as the receptors and downstream mediators that are required for RSPO-dependent signaling. These data suggest that RSPOs may contribute to potentiation of Wnt/beta-catenin signaling through autocrine, cell-autonomous pathways once Ewing sarcoma cells are exposed to exogenous Wnt ligands. Although the expression of RSPO during development has been described, ${ }^{16}$ the expression patterns of these molecules in adult tissue has not been well-characterized. Thus, its potential as a mediator of metastasis in the metastatic niche remains unclear. In these studies, we have demonstrated that sites to which Ewing sarcoma frequently metastasize also express RSPOs. Expression of RSPOs in these tissues may 
contribute to homing of tumor cells to metastatic sites, engraftment of these cells, and/or maintenance of metastatic growth. In addition, our studies reveal that the expression profiles of RSPOs, LGR receptors, and RNF molecules are highly variable among different Ewing sarcoma cell lines and target metastatic tissues. As a result, differences in these expression patterns may confer different sensitivities to Wnt/beta-catenin signaling in response to RSPO ligands. Moreover, new evidence supports roles for RSPOs in normal development that are Wnt-independent. ${ }^{17}$ The downstream impact of RSPO expression and activation on downstream signaling and tumor phenotypes in the context of Ewing sarcoma warrants further investigation.

These early findings that anti-RSPO2 therapy may decrease the incidence of metastases provide evidence that the Wnt/beta-catenin pathway may be amenable to therapeutic targeting in Ewing sarcoma. In further support of this, a recent xenograft study of a porcupine inhibitor showed enhanced metastasis-free survival in mice with orthotopic bone tumors that received the Wntpathway inhibitor. ${ }^{7}$ Despite the modest reduction of Wnt reporter activity that was observed upon administration of the anti-RSPO2 monoclonal antibody in vitro, we observed a significant reduction in the number of metastatic nodules that formed in vivo in a xenograft model of spontaneous metastasis. Given our data, we posit that the enhanced impact of anti-RSPO2 treatment in vivo likely comes from neutralization of Rspo2 in the metastatic tissues rather than in Ewing sarcoma cells themselves. This is due to the fact that TC32 cells express only modest levels of the gene RSPO2, whereas Rspo2 is robustly expressed in tissues that are targets of metastasis. In particular, Rspo2 is expressed in the small intestine, which was a frequent site of metastatic spread in this model.

A major limitation of this study is that we only tested the effects of RSPO2 inhibition on metastasis using the subrenal capsule model with TC32 cells. Using other Ewing sarcoma cells that express higher levels of RSPO2 (eg, A673 or CHLA32) may lead to more dramatic results. Further, investigation of the role of other RSPOs is warranted, especially given that RSPO3 is robustly expressed by the highly metastatic cell line TC32, making it a promising target for investigation.

In both subcutaneous and renal capsule models, administration of the anti-RSPO2 antibody had no impact on growth of primary tumors. Given that RSPO2/Wnt/beta-catenin signaling has little effect on proliferation of Ewing sarcoma cells, ${ }^{4}$ it was not unexpected that pharmacologic modulation of this pathway via RSPO inhibition had no effect on tumor size. This is because in vitro Ewing sarcoma cell-line proliferation, in general, correlates with in vivo tumor size. ${ }^{118}$ Further, although RSPO2 inhibition decreased the incidence of metastatic nodules, when metastatic tumors did grow in antiRSPO2 treated mice, they were often comparable in size to control tumors, suggesting that RSPO2 inhibition has an effect on seeding but not outgrowth of metastatic tumors once established. Despite this, the overall decrease in the number of metastatic foci emphasizes that tumor size is not the only therapeutic outcome that can and should be assessed in preclinical studies of efficacy, particularly due to the observation that the number of metastases and the number of sites of metastasis correlate with prognosis in many tumors, including Ewing sarcoma. ${ }^{19-22}$

The reduction of metastatic seeding by this single agent is noteworthy, especially considering the modest impact that RSPO2 inhibition had on overall Wht activation. In future studies, it will be worth determining whether combination therapy using anti-RSPO antibodies and inhibitors of canonical Wht ligand/receptor interaction, such as monoclonal antibody OMP-18R5,23-25 could result in even greater inhibition of metastatic burden. Together, these data and our previous work provide compelling evidence to support further preclinical investigation of anti-RSPO/Wnt/ 
beta-catenin approaches for metastasis inhibition in Ewing sarcoma.

\section{Conclusion}

This work characterizes the expression pattern of RSPOs and its associated signaling molecules in Ewing sarcoma. RSPO2 contributes to Ewing sarcoma metastasis and may be amenable as a target to therapeutically inhibit metastatic foci formation.

\section{Acknowledgments}

This work was supported by the SARC Sarcoma SPORE Grant U54 CA168512 from the National Institutes of Health $(\mathrm{NIH})$, an Alex's Lemonade Stand Foundation Innovation Award, and a Ruth L. Kirschstein National Research Service Award (NRSA) F30CA183276 (E.A. Pedersen) from the National Cancer Institute (NCI). Additional support was provided by the University of Michigan's Cancer Center Support Grant (P30 CA046592) through the use of the following Cancer Center core(s): flow cytometry, cellular imaging, and vector.

\section{References}

1. Lawlor ER, Sorensen PH. Twenty years on: what do we really know about Ewing sarcoma and what is the path forward? Crit Rev Oncog. 2015;20(3-4):155-171.

2. Balamuth NJ, Womer RB. Ewing's sarcoma. Lancet Oncol. 2010;11(2):184-192.

3. Pedersen EA, Menon R, Bailey KM, et al. Activation of Wnt/beta-catenin in Ewing sarcoma cells antagonizes EWS/ETS function and promotes phenotypic transition to more metastatic cell states. Cancer Res. 2016;76(17):5040-5053.

4. Scannell CA, Pedersen EA, Mosher JT, et al. LGR5 is expressed by Ewing sarcoma and potentiates Wnt/ beta-catenin signaling. Front Oncol. 2013;3:81.

5. Uren A, Wolf $\mathrm{V}$, Sun YF, Azari A, Rubin JS, Toretsky JA. Wnt/Frizzled signaling in Ewing sarcoma. Pediatr Blood Cancer. 2004;43(3):243-249.

6. Pridgeon MG, Grohar PJ, Steensma MR, Williams BO. Wht Signaling in Ewing sarcoma, osteosarcoma, and malignant peripheral nerve sheath tumors. Curr Osteoporos Rep. 2017;15(4):239-246.

7. Hayashi M, Baker A, Goldstein SD, et al. Inhibition of porcupine prolongs metastasis free survival in a mouse xenograft model of Ewing sarcoma. Oncotarget. 2017;8(45):78265-78276.

8. Nusse R, Clevers H. Wnt/beta-catenin signaling, disease, and emerging therapeutic modalities. Cell. 2017;169(6):985-999.

9. Schuijers J, Clevers H. Adult mammalian stem cells: the role of Wnt, Lgr5 and R-spondins. EMBO J. 2012;31(12):2685-2696.

10. Ruffner $\mathrm{H}$, SprungerJ, Charlat O, etal. R-spondin potentiates Wnt/beta-catenin signaling through orphan receptors LGR4 and LGR5. PLoS One. 2012;7(7): e40976. doi: 10.1371/journal.pone.0040976

11. Han T, Schatoff EM, Murphy C, et al. R-spondin chromosome rearrangements drive Wnt-dependent tumour initiation and maintenance in the intestine. Nat Commun, 2017;8:15945. doi: 10.1038/ncomms15945

12. Yin $X$, Yi H, Wang L, Wu W, Wu X, Yu L. R-spondin 2 promotes proliferation and migration via the Wnt/ beta-catenin pathway in human hepatocellular carcinoma. Oncol Lett. 2017;14(2):1757-1765.

13. Chartier C, Raval J, Axelrod F, et al. Therapeutic targeting of tumor-derived R-spondin attenuates beta-catenin signaling and tumorigenesis in multiple cancer types. Cancer Res. 2016;76(3):713-723.

14. Van Noord R, Thomas T, Krook M, et al. Tissuedirected implantation using ultrasound visualization for development of biologically relevant metastatic tumor xenografts. In Vivo. 2017;31(5):779-791.

15. Fuerer C, Nusse R. Lentiviral vectors to probe and manipulate the Wnt signaling pathway. PLoS One. 2010;5(2):e9370. doi: 10.1371/journal.pone.0009370.

16. Nam JS, Turcotte TJ, Yoon JK. Dynamic expression of R-spondin family genes in mouse development. Gene Expr Patterns. 2007;7(3):306-312.

17. Yan KS, Janda CY, Chang Jet al. Non-equivalence of Wht and R-spondin ligands during Lgr5+ intestinal stem-cell self-renewal. Nature. 2017;545(7653):238-242.

18. Svoboda LK, Bailey N, Van Noord RA et al. Tumorigenicity of Ewing sarcoma is critically dependent on the trithorax proteins MLL1 and menin. Oncotarget. 2017;8(1):458-471.

19. Khanna N, Pandey A, Bajpai J. Metastatic Ewing's sarcoma: revisiting the "evidence on the fence." Indian J Med Paediatr Oncol. 2017;38(2):173-181. 
20. Abdel-Rahman O. Clinical correlates and prognostic value of different metastatic sites in metastatic renal cell carcinoma. Future Oncol. 2017. doi: 10.2217/fon-2017-0175.

21. Abdel-Rahman O. Clinical correlates and prognostic value of different metastatic sites in patients with malignant melanoma of the skin: a SEER database analysis. J Dermatolog Treat. 2018(2):176-181.

22. Cho JH, Kim S, Namgung $M$, et al. The prognostic importance of the number of metastases in pulmonary metastasectomy of colorectal cancer. World J Surg Oncol. 2015;13: 222.
23. Kahn M. Can we safely target the WNT pathway? Nat Rev Drug Discov. 2014;13(7):513-532.

24. Smith DC, Rosen LS, Chugh, R, et al. First-in-human evaluation of the human monoclonal antibody vantictumab (OMP-18R5; anti-Frizzled) targeting the WNT pathway in a phase I study for patients with advanced solid tumors. J Clin On Abstr. 2013;31(2540): 2540-2540.

25. Gurney A, Axelrod F, Bond CJ, et al. Wht pathway inhibition via the targeting of Frizzled receptors results in decreased growth and tumorigenicity of human tumors. Proc Natl Acad Sci (USA). 2012;109(29):11717-11722. 